\title{
One Application of the Quantized Electromagnetic Field outside the High-Dimensional Static Gauss-Bonnet Black Holes
}

\author{
Ming Zhang \\ Faculty of Science, Xi'an Aeronautical University, Xi'an, China \\ Email: zhangming@xaau.edu.cn
}

How to cite this paper: Zhang, M. (2018) One Application of the Quantized Electromagnetic Field outside the High-Dimensional Static Gauss-Bonnet Black Holes. Journal of Modern Physics, 9, 1785-1792. https://doi.org/10.4236/jmp.2018.99112

Received: July 19, 2018

Accepted: August 5, 2018

Published: August 8, 2018

Copyright $\odot 2018$ by author and Scientific Research Publishing Inc. This work is licensed under the Creative Commons Attribution International License (CC BY 4.0).

http://creativecommons.org/licenses/by/4.0/

\begin{abstract}
In present paper, we calculated the response rate of a static charge outside $d$-dimensional Gauss-Bonnet black hole in the low-frequency regime, by using the quantization of an electromagnetic field in the background of static spherically symmetric $d$-dimensional spacetime in the Boulware vacuum, which can be expressed as the summation of hypergeometric functions.
\end{abstract}

\section{Keywords}

Response Rate, Gauss-Bonnet Black Hole, High-Dimensional Spacetime

\section{Introduction}

An important prediction in this field is the thermal evaporation of black hole [1]. This nontrivial effect was soon realized to be closely associated with the existence of an event horizon in Schwarzschild spacetime. One of the difficulties in studying fields in Schwarzschild [2] and other black hole spacetime, even when the fields are non-interacting, stems from the fact that the solutions to the field equations are functions whose properties are not well known. In the low-frequency regime, however, the situation is much simpler and the mode functions of the massless scalar field are well known [3]. Recently, Crispino et al. [4] suggested a scheme to quantize the free quantum electrodynamics in static spherically symmetric $d$-dimensional spacetime and gave out the response rate of a static charge outside the four-dimensional Schwarzschild black hole.

Following the advent of string theory, extra dimensions were promoted from an interesting curiosity to a theoretical necessity since superstring theory 
requires an eleven-dimensional spacetime to be consistent from a quantum point of view ([5] [6] [7] [8]). Among the higher curvature gravities, the most extensively studied theory is the so-called Gauss-Bonnet gravity ([9]-[15]), which naturally emerges when we want to generalize Einstein's theory in higher dimensions by keeping all characteristics of usual general relativity excepting the linear dependence of the Riemann tensor. Therefore, it is necessary to study the quantization of the free electromagnetic field with the high-dimensional spacetime and its specific application outside a Gauss-Bonnet black hole.

In this paper, under the quantized electromagnetic field, we calculate the response rate of a static charge outside the the $d$-dimensional GB black hole in the Unruh vacuum [16]. The response rate is a quantum concept with no natural analog in classical physics though it is possible to define a corresponding classical quantity mathematically and represents the number of times the source responds to the field per unit time.

The paper is organized as follows. In Section 2, we review the basic concepts of the electromagnetic field in the arbitrary dimensional spacetime of a spherically symmetric black hole in a modified Feynann gauge. Section 3 devotes to calculate the response rate of a static charge outside a GB black hole of arbitrary-dimensions. In Section 5, we summarize the main results.

\section{Gupta-Bleuler Quantization in a Modified Feynman Gauge}

In this section, we follow the notation of Ref. [4] to study the solutions of field equations for electromagnetic field in an asymptotic flat and static spherically symmetric $(p+2)$-dimensional spacetime. The quantization of electromagnetic field will be carried out in the frame of Gupta-Bleuler formalism in a modified Feynmann gauge.

The line element under considered takes the form

$$
\mathrm{d} \tau^{2}=f(r) \mathrm{d} t^{2}-h(r) \mathrm{d} r^{2}-r^{2} \mathrm{~d} s_{p}^{2}
$$

with the line element of a unit $p$-sphere $\mathrm{d} s_{p}^{2}$. We assume that both $f(r)$ and $h(r)^{-1}$ have a zero at $r=r_{h}$ and positive for $r>r_{h}$.

The Lagrangian density for electromagnetic field in a modified Feynman gauge is

$$
\mathcal{L}_{F}=\sqrt{-g}\left[-\frac{1}{4} F_{\mu v} F^{\mu v}-\frac{1}{2} G^{2}\right],
$$

and $G$ stands for the modified Feynman gauge

$$
G=\nabla^{\mu} A_{\mu}+K^{\mu} A_{\mu} .
$$

Here the vector $K^{\mu}$ is independent on electromagnetic field $A_{\mu}$, and takes the form

$$
K^{\mu}=\left(0, f^{\prime} /(f h), 0,0\right) .
$$

Under this choice, the gauge condition changes into 


$$
G=\frac{1}{f} \partial_{t} A_{t}-\sqrt{\frac{f}{h}} \frac{1}{r^{p}} \partial_{r}\left[\frac{r^{p}}{\sqrt{f h}} A_{r}\right]-\frac{1}{r^{2}} \nabla^{i} A_{i}
$$

From the Lagrangian density for electromagnetic field, the equations of motion are

$$
\left\{\begin{array}{l}
-\frac{1}{f} \partial_{t}^{2} A_{t}+\sqrt{\frac{f}{h}} \frac{1}{r^{p}} \partial_{r}\left[\frac{r_{p}}{\sqrt{f h}} \partial_{r} A_{r}\right]+\frac{1}{r^{2}} \nabla^{2} A_{t}=0 \\
-\frac{1}{f} \partial_{t}^{2} A_{r}+\frac{1}{f} \partial_{r}\left[\sqrt{\frac{f}{h}} \frac{f}{r^{p}} \partial_{r}\left(\frac{r_{p}}{\sqrt{f h}} A_{r}\right)\right]+\frac{1}{r^{2}} \nabla^{2} A_{r}+\frac{1}{f} \partial_{r}\left(\frac{f}{r^{2}}\right) \nabla^{i} A_{i}=0 \\
-\frac{1}{f} \partial_{t}^{2} A_{i}+\frac{r^{2-p}}{\sqrt{f h}} \partial_{r}\left(\sqrt{\frac{f}{h}} r^{p-2} \partial_{r} A_{i}\right)-\frac{r^{2}}{f h} \partial_{r}\left(\frac{f}{r^{2}}\right) \partial_{i} A_{r} \\
+\frac{1}{r^{2}}\left[\nabla^{j}\left(\nabla_{j} A_{i}-\nabla_{i} A_{j}\right)+\partial_{i}\left(\nabla^{j} A_{j}\right)\right]=0, \quad(j=1, \cdots, p)
\end{array}\right.
$$

Here $\nabla_{i}$ is the covariant derivative on $S^{p}$.

We denote the complete set of solutions of Equation (6) by $A_{\mu}^{(\lambda n ; \omega l m)}$, and call a non-physical modes for $\lambda=0$, physical modes for $\lambda=1,2,3, \cdots, p$ and a pure-gauge mode for $\lambda=p+1$. The label $n$ represents modes incoming from the past null infinity $(n=\leftarrow)$ and coming out from the past horizon $(n=\rightarrow)$.

\subsection{Physical Modes}

For other independent solutions $\lambda=1,2, \cdots, p$, which represent physical degrees of freedom, the time-component can be taken as zero. They are the linear independent solution of Equation (6) with a gauge condition $G=0$, and are classified into two types.

Physical Modes 1

The "physical modes 1" solution can be written as [4]

$$
\left\{\begin{array}{l}
A_{t}^{(1 n ; \omega l m)}=0 \\
A_{r}^{(1 n ; \omega l m)}=R_{\omega l}^{(1 n)}(r) Y_{l m} \mathrm{e}^{-i \omega t},(l \geq 1) \\
A_{i}^{(1 n ; \omega l m)}=\frac{r^{2-p}}{l(l+p-1)} \sqrt{\frac{f}{h}} \frac{\mathrm{d}}{\mathrm{d} r}\left[\frac{r^{p}}{\sqrt{f h}} R_{\omega l}^{(1 n)}(r)\right] \partial_{i} Y_{l m} \mathrm{e}^{-i \omega t}
\end{array}\right.
$$

where $i=1,2,3, \cdots, p$ and:

$$
\left[\frac{\omega^{2}}{f}-\frac{l(l+p-1)}{r^{2}}\right] R_{\omega l}^{(1 n)}(r)+\frac{1}{r^{2}} \frac{\mathrm{d}}{\mathrm{d} r}\left[\sqrt{\frac{f}{h}} r^{2-p} \frac{\mathrm{d}}{\mathrm{d} r}\left(\frac{r^{p}}{\sqrt{f h}} R_{\omega l}^{(1 n)}(r)\right)\right]=0
$$

\subsection{Quantization}

Using Gupta-Bleuler quantization, we impose the equal-time commutation relations on the field $\hat{A}_{\mu}$ and momentum $\hat{\Pi}^{t \mu}$ operators

$$
\begin{gathered}
{\left[\hat{A}_{\mu}(t, \mathbf{x}), \hat{A}_{v}\left(t, \mathbf{x}^{\prime}\right)\right]=\left[\hat{\Pi}^{t \mu}(t, \mathbf{x}), \hat{\Pi}^{t v}\left(t, \mathbf{x}^{\prime}\right)\right]=0} \\
{\left[\hat{A}_{\mu}(t, \mathbf{x}), \hat{\Pi}^{t v}\left(t, \mathbf{x}^{\prime}\right)\right]=\frac{i \delta_{\mu}^{v}}{\sqrt{-g}} \delta^{p+1}\left(\mathbf{x}-\mathbf{x}^{\prime}\right)}
\end{gathered}
$$


where $\mathbf{x}$ and $\mathbf{x}^{\prime}$ represent all spatial coordinates. The field $\hat{A}_{\mu}$ can be expressed in terms of $A_{\mu}^{(\xi)}$

$$
\hat{A}_{\mu}(t, \mathbf{x})=\sum_{\rho} \int_{-\infty}^{+\infty} \frac{\mathrm{d} \omega}{\sqrt{4 \pi|\omega|}} A_{\mu}^{(\omega \rho)}(t, \mathbf{x}) a_{\omega \rho},
$$

where $A_{\mu}^{(-\omega \rho)} \equiv \overline{A_{\mu}^{(\omega \rho)}}, \quad a_{-\omega \rho} \equiv a_{\omega \rho}^{\dagger}$ and $\rho$ labels all quantum numbers.

\section{Response Rate of a Static Charge Outside a D-Dimensional Gauss-Bonnet Gravity Black Hole}

In this section, we will calculate the response rate of a static charge outside a $d$-dimensional $(d=p+2)$ Gauss-Bonnet black hole by following the procedure of Ref. [4]. In this case, the black hole is characterized by $f(r)=h(r)^{-1}=1-\left(r_{h} / r\right)^{(p-1)}$.

The action of pure GB can be written as

$$
S=\int \mathrm{d}^{p+2} x \sqrt{-g} \alpha \mathcal{L}_{2}
$$

where the coupling constant $\alpha$ can be regarded as the inverse of string tension and be assumed $\alpha>0$ in this paper. The Gauss-Bonnet term is given by

$$
\mathcal{L}_{2}=R^{2}-4 R_{a b} R^{a b}+R_{a b c d} R^{a b c d} .
$$

The line element for the exterior region of the $d$-dimensional Gauss-Bonnet gravity is given by

$$
\mathrm{d} s^{2}=f(r) \mathrm{d} t^{2}-f(r)^{-1} \mathrm{~d} r^{2}-r^{2} \mathrm{~d} s_{p}^{2}
$$

The $f(r)$ in the $d$-dimensional GB gravity is

$$
f(r)=1-\sqrt{\frac{2 M}{\alpha r^{d-5}}} \equiv 1-\frac{B}{r^{\frac{d-5}{2}}},
$$

The horizon radius $r_{h}=\left(\frac{2 M}{\alpha}\right)^{\frac{1}{d-5}}=B^{\frac{2}{d-5}}$.

In order to avoid the indefinite results ([17] [18]), we use the formula suggested by Crispino et al. [19] and assume the static charge located at $\left(r_{0}, \theta_{0}\right)$ with a current density $j^{\mu}$

$$
\left\{\begin{array}{l}
j^{\mu}=\left(j^{t}, j^{r}, 0, \cdots, 0\right) \\
j^{t}=\frac{\sqrt{2} q \cos E t}{\sqrt{-g}} \delta\left(r-r_{0}\right) \delta\left(\theta_{1}-\theta_{10}\right) \cdots \delta\left(\theta_{p}-\theta_{p 0}\right) \\
j^{r}=\frac{\sqrt{2} q E \sin E t}{\sqrt{-g}} \Theta\left(r-r_{0}\right) \delta\left(\theta_{1}-\theta_{10}\right) \cdots \cdots \delta\left(\theta_{p}-\theta_{p 0}\right)
\end{array}\right.
$$

The step function $\Theta(x)$ is defined by $\Theta(x)=1,(x>0)$ and vanishing for $x \leq 0$.

Such current interacts with vector potential $A_{\mu}$ through the Lagrangian $\sqrt{-g} j^{\mu} A_{\mu}$. Since $a_{\omega l m}^{((p+1) n) \dagger} \mid$ phys $\rangle$ is non-physical states, which excludes the interaction with the pure-gauge particles created by $a_{\omega l m}^{((p+1) n) \dagger}$. We will neglect it. 
However, the current does interact with the states created by $a_{\omega l m}^{(0 n) \dagger}$ but the contribution to physical probabilities maybe taken as zero once the non-physical modes are appropriately chosen. Furthermore, there is no interacting term between the current and physical modes 2 due to $A_{t}=A_{r}=0$. Therefore, we only need to consider the physical modes 1 . To make the counting process more concise, we will limit in the spherical coulomb gauge. The mode function can be written as [4]

$$
\begin{gathered}
A_{t}^{1^{1} n ; \omega l m}=\frac{i \omega r^{2-p}}{l(l+p-1)} \sqrt{\frac{f}{h}} \frac{\mathrm{d}}{\mathrm{d} r}\left(\frac{r^{p}}{\sqrt{f h}} R_{\omega l}^{1 n}\right) Y_{l m} \mathrm{e}^{-i \omega t} \\
A_{r}^{1^{\prime} n ; \omega l m}=\frac{\omega^{2} r^{2}}{l(l+p-1)} \frac{1}{f} R_{\omega l}^{1 n} Y_{l m} \mathrm{e}^{-i \omega t} .
\end{gathered}
$$

In the limit $E \rightarrow 0$, the proper response rate of the charge can be written [4]

$$
\frac{R_{0 l m}}{\sqrt{f\left(r_{0}\right)}}=4 \pi \lim _{E \rightarrow 0} \frac{\left|\mathcal{T}_{E l m}\right|^{2}}{\sqrt{f\left(r_{0}\right)} \beta E}
$$

where $\mathcal{T}_{E l m}$ is the index $n=\rightarrow$ in transition amplitude $\mathcal{T}_{\text {olm }}^{n}$ which has the form

$$
\mathcal{T}_{\omega l m}^{n} \equiv \frac{1}{2 \pi \delta(\omega-E)} \int \mathrm{d}^{p+2} x \sqrt{-g} j^{\mu}\left\langle 1 n ; \omega l m\left|\hat{A}_{\mu}\right| 0\right\rangle
$$

Now, we return to calculate $\mathcal{T}_{E l m}^{\rightarrow}$. The Equation (8) can be written

$$
\frac{1}{r^{2}} \frac{\mathrm{d}}{\mathrm{d} r}\left[f r^{2-p} \frac{\mathrm{d}}{\mathrm{d} r}\left(r^{p} R_{\omega l}^{(1 \rightarrow)}(r)\right)\right]-\frac{l(l+p-1)}{r^{2}} R_{\omega l}^{(1 \rightarrow)}(r)+\frac{\omega^{2}}{f} R_{\omega l}^{(1 \rightarrow)}(r)=0 .
$$

After introducing the Wheeler tortoise coordinate and a function $\varphi$

$$
R_{\omega l}^{(1 \rightarrow)}(r) \equiv \frac{\sqrt{l(l+p-1)}}{\omega} r^{-\frac{p}{2}-1} \varphi_{\omega l}^{(1 \rightarrow)}(r),
$$

the Equation (21) changes into

$$
\left(\omega^{2}+\frac{\mathrm{d}^{2}}{\mathrm{~d} r^{* 2}}-V_{1}\left(r^{*}\right)\right) \varphi_{\omega l}^{(1 \rightarrow)}(r)=0
$$

with

$$
V_{1}\left[r^{*}(r)\right]=f \frac{l(l+p-1)}{r^{2}}+f^{2} \frac{p(p-2)}{4 r^{2}}-f^{\prime} \frac{(p-2)}{2 r}
$$

For the small $\omega$ and the condition $\left(r-r_{h} \ll \omega^{2} r_{h}^{3},\left|\omega r^{*}\right| \ll 1\right)$, the wave coming from the past horizon $H^{-}$is almost completely reflected back by the potential toward the horizon

$$
\varphi_{\omega l}^{(1 \rightarrow)} \approx-2 \omega r^{*}+\text { const }
$$

Generally, it is hard to find the analytic expression for the Wheeler tortoise coordinate $r^{*}$. Fortunately, what we need is just the behavior of $r^{*}$ near horizon. Substituting the expression of $f(r)$, the leading term of the Wheeler tortoise coordinate can be written 


$$
r^{*} \approx \frac{2}{p-3}\left(\frac{2 M}{\alpha}\right)^{\frac{1}{p-3}} \ln (z-1)
$$

by using the transition

$$
z=\frac{2}{B} r^{\frac{p-3}{2}}-1
$$

The boundary condition of $R_{\omega l}^{(1 \rightarrow)}$ reads

$$
R_{\omega l}^{(1 \rightarrow)} \approx-\frac{\sqrt{l(l+p-1)}}{p-3} 2^{\frac{3 p-4}{p-3}} B^{-\frac{p}{p-3}} \ln (z-1),\left(r-r_{H} \ll \omega^{2} r_{H}^{3},\left|\omega r^{*}\right| \ll 1\right)
$$

In terms of variable $z$, Equation (21) can be written

$$
\begin{aligned}
& \frac{1}{4}\left(z^{2}-1\right) \frac{\mathrm{d}^{2} q(z)}{\mathrm{d} z^{2}}+\frac{1}{4}\left[\frac{3 p-1}{p-3}(z-1)+2\right] \frac{\mathrm{d} q(z)}{\mathrm{d} z} \\
& +\frac{1}{(p-3)^{2}}\left[-l(l+p-1)+\frac{p(p-5)}{z+1}+p+\omega^{2} \frac{z+1}{z-1}\left(\frac{B}{2}(z+1)\right)^{\frac{4}{p-3}}\right] q(z)=0
\end{aligned}
$$

and this equation can be solved explicitly for small $\omega$ limit. Combining the asymptotic behavior $R_{\omega l}^{(1 \rightarrow)} \rightarrow 0$ as $z \rightarrow+\infty$, the solution of Equation (29) is

$$
\begin{aligned}
R_{\omega l}^{(1 \rightarrow)}= & \frac{\sqrt{l(l+p-1)}}{p-3} 2^{\frac{3 p-4}{p-3}} B^{-\frac{p}{p-3}} \\
& \times \mathrm{F}\left(\left[\frac{2 l}{p-3}, \frac{-2 l-2 p+2}{p-3}\right],\left[\frac{-2 p+2}{p-3}\right], \frac{z+1}{2}\right)(z+1)^{-\frac{2 p}{p-3}}
\end{aligned}
$$

where the coefficient has been appropriately chosen to agreement with the boundary condition Equation (28).

Thus, the expression of the proper response rate of the charge is

$$
\frac{R_{0 l m}}{\sqrt{f\left(r_{0}\right)}}=\frac{(p-3) q^{2}\left(z_{0}+1\right)^{-\frac{2 p+2}{p^{-3}}} B^{\frac{2-2 p}{p-3}} 2^{\frac{6}{p-3}}}{\pi l(l+p-1) \sqrt{f\left(r_{0}\right)}}\left(\frac{z_{0}-1}{z_{0}+1}\right)^{2}\left[\frac{\mathrm{d}}{\mathrm{d} z_{0}} \mathrm{~F}_{l}\left(z_{0}\right)\right]^{2}\left|Y_{l m}\right|^{2}
$$

ubstituting the all parameters for $d$-dimensional GB black hole

$$
\begin{gathered}
z_{0}=\frac{2}{B} r_{0}^{\frac{p-3}{2}}-1 \\
f\left(r_{0}\right)=1-\frac{B}{r_{0}^{\frac{d-5}{2}}} \\
\mathrm{~F}_{l}\left(z_{0}\right)=\mathrm{F}\left(\left[\frac{2 l}{p-3}, \frac{-2 l-2 p+2}{p-3}\right],\left[\frac{-2 p+2}{p-3}\right], \frac{z+1}{2}\right)
\end{gathered}
$$

the total transition probability per proper time of the charge is given by

$$
\begin{aligned}
R^{\mathrm{tot}} & =\sum_{l}^{+\infty} \sum_{m=-1}^{l} \frac{R_{0 l m}}{\sqrt{f\left(r_{0}\right)}} \\
& =\sum_{l}^{+\infty} \frac{(p-3) q^{2}\left(z_{0}+1\right)^{-\frac{2 p+2}{p-3}} B^{\frac{2-2 p}{p-3}} 2^{\frac{6}{p-3}}}{\pi l(l+p-1) \sqrt{f\left(r_{0}\right)}}\left(\frac{z_{0}-1}{z_{0}+1}\right)^{2}\left[\frac{\mathrm{d}}{\mathrm{d} z_{0}} \mathrm{~F}_{l}\left(z_{0}\right)\right]^{2} \frac{G(l)}{\Omega_{p}}
\end{aligned}
$$


We can give the main contribution of the result of the Schwarzschild black hole and GB black hole,

$$
\begin{gathered}
R^{\mathrm{tot}} \sim\left(\frac{r_{h}}{r_{0}}\right)^{p-3},(\text { Schwarzschild }) \\
R^{\mathrm{tot}} \sim\left(\frac{r_{h}}{r_{0}}\right)^{\frac{3 p-1}{2}} \cdot(\mathrm{GB})
\end{gathered}
$$

Assuming that there is a charge outside an unknown black hole, the radius of the horizon $\left(r_{h}\right)$, the mass of the black hole $(M)$ and the location of the charge $\left(r_{0}\right)$ are known. Meanwhile, we can measure the response rate of the charge outside this unknown black hole. Compare the measured value with Equation (36) and Equation (37), then we can tell the black hole is the GB type or not.

\section{Conclusions}

In this paper, we quantized the free electrodynamics in static spherically symmetric spacetime of arbitrary dimensions in a modified Feynman gauge. Then we examined the Gupta-Bleuler quantization in this modified Feynman gauge. The results obtained were applied to compute the total response rate of a static charge outside the $d$-dimensional GB black hole in the Unruh vacuum.

For the Einstein-Gauss-Bonnet gravity, one can follow the same procedure. The Wheeler tortoise coordinate $r^{*}$ has a asymptotic behavior $r^{*} \approx f^{\prime}\left(r_{h}\right)^{-1} \ln \left(r-r_{h}\right)$ as near horizon. The boundary condition Equation (25) changes into $\varphi_{\omega l}^{(1 \rightarrow)} \approx-2 \omega / f^{\prime}\left(r_{h}\right) \ln \left(r-r_{h}\right)$. One problem is that we do not find a new variable to simplify the Equation (21) and its analytic solution. An applicable way is to find a series solution of Equation (21), the results can not be expressed in terms of familiar special functions and we neglect it here.

Such an outcome is not only a simple promotion work for what Crispino et al. [4] have done. Having the specific form of the free quantum electrodynamics in static spherically symmetric spacetime of arbitrary dimensions, It may provide us a chance for further investigating quantum field theory in high-dimensional curved spacetime. For instance, some authors ([19] [20] [21]) have researched whether or not a quantum version of the equivalence principle could be formulated and show some equivalence for low-frequency quantum phenomena in flat and curved spacetime. The same problem could be reconsidered in high-dimensional spacetime and discuss the dimension dependence of the results.

\section{Acknowledgements}

This work has been supported by the Scientific Research Program Funded by Shaanxi Provincial Education Department under Program No.16JK1394.

\section{Conflicts of Interest}

The authors declare no conflicts of interest regarding the publication of this paper. 


\section{References}

[1] Hawking, S.W. (1975) Communications in Mathematical Physics, 43, 199. [(1976) Erratum-Ibid, 46, 206]. https://doi.org/10.1007/BF02345020

[2] Jensen, B.P. and Candelas, P. (1986) Physical Review D, 33, 1590. [(1987) Erratum-Ibid, D35, 4041]. https://doi.org/10.1103/PhysRevD.33.1590

[3] Candelas, P. (1980) Physical Review D, 21, 2185. https://doi.org/10.1103/PhysRevD.21.2185

[4] Crispino, L.C.B., Higuchi, A. and Matsas, G.E.A. (2001) Physical Review D, 63, 124008. [(2009) Erratum-Ibid, D80, 029906]. https://doi.org/10.1103/PhysRevD.63.124008

[5] Horava, P. and Witten, E. (1996) Nuclear Physics B, 475, 94 [hep-th/9603142]. https://doi.org/10.1016/0550-3213(96)00308-2

[6] Lukas, A., Ovrut, B.A. and Waldram, D. (1999) Physical Review D, 60, 086001 [hep-th/9806022]. https://doi.org/10.1103/PhysRevD.60.086001

[7] Randall, L. and Sundrum, R. (1999) Physical Review Letters, 83, 3370 [hep-ph/ 9905221]. https://doi.org/10.1103/PhysRevLett.83.3370

[8] Randall, L. and Sundrum, R. (1999) Physical Review Letters, 83, 4690 [hep-th/ 9906064]. https://doi.org/10.1103/PhysRevLett.83.4690

[9] Lanczos, C. (1938) Annals of Mathematics, 39, 842. https://doi.org/10.2307/1968467

[10] Lovelock, D. (1971) Journal of Mathematical Physics, 12, 498. https://doi.org/10.1063/1.1665613

[11] Zou, D.-C., Yang, Z.-Y., Yue, R.-H. and Yu, T.-Y. (2011) Chinese Physics B, 20, 100403 [arXiv:1107.2741 [gr-qc]]. https://doi.org/10.1088/1674-1056/20/10/100403

[12] Yue, R.-H., Zou, D.-C., Yu, T.-Y. and Yang, Z.-Y. (2011) Chinese Physics B, 20, 050401 [arXiv:1107.2743 [gr-qc]].

[13] Zhou, K., Yang, Z.-Y., Zou, D.-C. and Yue, R.-H. (2012) Chinese Physics B, 21, 020401 [arXiv:1107.2732 [gr-qc]]. https://doi.org/10.1088/1674-1056/21/2/020401

[14] Boulware, D.G. and Deser, S. (1985) Physical Review Letters, 55, 2656. https://doi.org/10.1103/PhysRevLett.55.2656

[15] Wheeler, J.T. (1986) Nuclear Physics B, 273, 732. https://doi.org/10.1016/0550-3213(86)90388-3

[16] Unruh, W.G. (1976) Physical Review D, 14, 870. https://doi.org/10.1103/PhysRevD.14.870

[17] Higuchi, A., Matsas, G.E.A. and Sudarsky, D. (1992) Physical Review D, 45, 3308. https://doi.org/10.1103/PhysRevD.45.R3308

[18] Higuchi, A., Matsas, G.E.A. and Sudarsky, D. (1992) Physical Review D, 46, 3450. https://doi.org/10.1103/PhysRevD.46.3450

[19] Crispino, L.C.B., Higuchi, A. and Matsas, G.E.A. (1998) Physical Review D, 58, 084027 [gr-qc/9804066]. https://doi.org/10.1103/PhysRevD.58.084027

[20] Candelas, P. and Sciama, D.W. (1983) Physical Review D, 27, 1715. https://doi.org/10.1103/PhysRevD.27.1715

[21] Kleinert, H. (1996) Quantum Equivalence Principle. Cargese, Functional Integration, 67-92 [Quant-ph/9612040]. 